\title{
Un essai de Wieland Wagner sur le théâtre adressé à Paul Boulet
}

\section{Philippe Olivier}

\section{Q OpenEdition}

1 Journals

Édition électronique

URL : https://journals.openedition.org/rbnu/2352

DOI : $10.4000 /$ rbnu. 2352

ISSN : 2679-6104

\section{Éditeur}

Bibliothèque nationale et universitaire de Strasbourg

\section{Édition imprimée}

Date de publication : 1 mai 2013

Pagination : 64-73

ISBN : 9782859230432

ISSN : 2109-2761

\section{Référence électronique}

Philippe Olivier, "Un essai de Wieland Wagner sur le théâtre adressé à Paul Boulet », La Revue de la BNU [En ligne], 7 | 2013, mis en ligne le 01 mai 2013, consulté le 08 août 2021. URL : http:// journals.openedition.org/rbnu/2352; DOI : https://doi.org/10.4000/rbnu.2352

\section{(c) (i) (2)(2)}

La Revue de la BNU est mise à disposition selon les termes de la Licence Creative Commons Attribution - Pas d'Utilisation Commerciale - Partage dans les Mêmes Conditions 4.0 International. 
Depuls la reprise des festivals de Beyreuth on 1951 la discussion rolative aux possibilités ot aux linites d'une mise en scène moderne des oeuvres de Wacner a pris une violence telle qu'on pourra1t eroire qu'11 s'ag1t d'un problème brulant d'1nterprétation. Ie gujet de cotte discussion n'a pas varié $t$ les Indications scén1ques de Rlchard Wagner dolvent-elles être consiđérées, au mône t1tre que le poère et la musique, comme un élément Itmuable de I'oeuvre ? Et, en ce cas, le falt de s'écerter des "qnnotations 1ndirectes" équivaut-11 a 1 'abandon volontalre du systome entier ?

N'est-11 pas ourleux, en expminant les selze tomes que représentent les "Deuvres complates" de Rlchard Wagner et sa correspondence s'élevant a des milliers de lettres qu'11 ne mentianne point ce theme 1nsidieux ? In se devalt de gagner ses contemporains A son oeurre et s'est peu inquíté du sort que $I^{\prime a v e n i r-}$ 101 réservera1t.Les très rares notes euthent1ques a ce propos, Qu'on alne toujours a citer, concernent des cas particuliers et $n^{\prime} o n t$, de ce fa1t, pas une 1mportance génórale. th génie de la valeur de Wagner n'est pas doctrinalre. Ses rigoureuses adaptations d'oeuvres efassiques dont la forme originale nous parait inaltérable révalent a quel point 11 pensa1t sans cotaprom1s aux problames du théatre vivent . .

Io Bayreuth postwagnérien $\chi$, et surtout Hans Pritzner at ses disciples ont déclaré que les Indications scénlques de Wagner étalent sacrées et faisaient intégraloment pertie de son oeuvre. Le ton 1 rrité de Pritzner pour réclamer une zusel1ère nationale pour les cher's d'orchestre et netteurs en scane trop pleins de pensée personnelle, "nn đécret national pour la protection qes oeuvres d'ert contre leur chicat altération arbitraire par des instituts voús au service de I'art" n'est que la conséquence 


\section{Quand Paul Boulet attaquait Wieland Wagner et recevait en réponse un essai sur le théâtre}

A lors qu'approche, à Bayreuth, la Tétralogie dite " du bicentenaire $~^{1}$, dont la mise en scène sera confiée à l'Allemand Frank Castorf, le radical directeur de la Volksbühne de Berlin, les divergences opposant wagnériens conservateurs et amateurs progressistes d'art lyrique sont devenues un thème d'actualité récurrent, en tout cas dans des pays tels que la France et l'Italie. Ces conflits ne sont pas nouveaux. Ils existaient déjà lors de la saison inaugurale du Festival de Bayreuth en 1876, ont connu un pic un siècle après, lors du légendaire Ring signé Patrice Chéreau, et ont continué à défrayer la chronique quand Jürgen Flimm, Heiner Müller ou Christoph Schlingensief ont présenté leurs réalisations théâtrales sur la " verte colline".

Cette querelle entre conservateurs et novateurs atteignit aussi une dimension paroxystique au temps du " Neu-Bayreuth " : on désigne, par ce terme, la révolution esthétique qui s'y est produite entre 1951 et 1966 , l'année du décès de Wieland Wagner. L'entrée du fonds Boulet-Devraigne à la Bibliothèque nationale et universitaire de Strasbourg en porte témoignage. Une importante correspondance a en effet été échangée entre Paul Boulet et Wieland Wagner, ainsi qu'entre le wagnérien parisien et la redoutable Winifred, mère du même Wieland. Le haut fonctionnaire Paul Boulet, auquel une contribution de Mathieu Schneider est consacrée dans cette livraison, était tout sauf un inconnu parmi les milieux musicaux parisiens. Ainsi, le compositeur Henri Sauguet le classait parmi les zélateurs de Wagner " des plus purs et plus documentés ${ }^{2}$. Il devait, entre autres, être parmi les initiateurs d'une émission de télévision diffusée le 12 mars 1961 et consacrée au centenaire de la création de la version parisienne de Tannhäuser.

Fréquentant le Festival de Bayreuth depuis 1937, Paul Boulet s'y était aussi précipité dès l'été de sa réouverture effectuée en 1951. Après s'être apparemment réjoui du style de mise en scène adopté par Wieland ${ }^{3}$, il devint l'un de ses ennemis les plus implacables, n'hésitant pas, cependant, à verser des larmes de crocodile à la suite de son décès : "Wieland était un homme très intelligent et un véritable artiste " ${ }^{4}$. Ces propos de rigueur au long d'une correspondance de condoléances n'empêchèrent pas auparavant Boulet de s'en prendre au " caractère prétentieux et surtout obstiné $"{ }^{5}$ du défunt. Il lui adressa régulièrement des extraits d'articles parus dans la presse française, dont le ton hostile était représentatif de la personnalité de leurs signataires. À l'exception d'Antoine Goléa et, dans une moindre mesure, de Claude Rostand, des commentateurs comme Bernard Gavoty ou Jacques Rozner - le rédacteur en chef du quotidien économique Les Échos - sont tout sauf des foudres de 
modernité. Ils incarnent des valeurs profondément anachroniques. Ils s'en prendraient aujourd'hui à Jonathan Meese, chargé de mettre en scène Parsifal à Bayreuth en 2015.

Ainsi, la lettre adressée le 30 octobre 1963 par le Français au petit-fils du compositeur de Lohengrin constitue l'un des moments les plus ardents d'une confrontation d'arguments durant depuis une bonne décennie. Ayant renoncé à ses précédentes admonestations au ton paternaliste, Boulet menace Wieland de l'interpeller par voie de presse s'il ne répond pas à la question de savoir pourquoi il ne respecterait pas " les indications précises données par le Maître dans ses livrets pour la présentation scénique de ses œuvres lyriques ". À cet égard, le Français montre - comme à d'autres occasions - qu'il a parfaitement conscience du rôle déjà tenu par les médias dans la société d'alors. La réponse de Wieland, datée du 23 novembre de la même année, est cinglante : "Vous serez d'accord avec moi que notre correspondance concernant les détails de la présentation scénique des œuvres de Richard Wagner est très inféconde, car nous ne pourrons jamais nous accorder sur nos conceptions artistiques ", écrit-il à Boulet. Rappelons que l'été précédent avait donné lieu à une polémique intense au sujet de sa seconde production des Maîtres chanteurs de Nuremberg. Certains avaient vu en elle une tentative insolente de gommer le substrat germanique de l'œuvre.

Wieland joint à sa lettre une traduction française d'un de ses textes fameux, intitulé Wagner, monument historique ?, et qui constitue sa véritable réponse esthétique à Boulet. Ce texte, à notre connaissance, est jusqu'à aujourd'hui resté inédit en français. A l'époque de la correspondance entre les deux hommes, il venait de paraître dans un recueil d'articles consacrés au compositeur $^{6}$, et que Wieland avait dirigé à la demande de l'éditeur List. Il comprenait entre autres des textes de Hans Mayer, Theodor Adorno, Curt von Westernhagen et donc ce Denkmalsschutz für Wagner ?, où Wieland expose largement sa conception de la réception des classiques après leur mort.

Le caractère talmudique et répétitif des observations de Boulet, son obstination à reprendre les théories formulées en 1895 par Adolphe Appia dans l'un de ses essais ${ }^{7}$, sa désapprobation des éclairages intentionnellement parcimonieux et fort efficaces employés par Wieland ${ }^{8}$ l'amènent alors à comploter contre lui en échangeant des courriers avec sa propre mère... Wini- fred, la Lady Macbeth de Bayreuth. Non seulement ils s'adressent réciproquement des vœux au début de chaque année nouvelle, mais Boulet transmet, de surcroît, à Winifred la copie des courriers qu'il envoie à Wieland! Leur objectif commun est de pulvériser le "Neu-Bayreuth ". La veuve de Siegfried aurait connu Paul Boulet depuis 1937. Ils gravitent tous deux dans un cercle de relations ayant profité de leur proximité avec les maîtres du Troisième Reich - quand bien même elles n'ont pas approuvé les horreurs perpétrées en son nom. Y figurent la cantatrice Germaine Lubin, dont la naïveté s'avérera fatale pour elle, le pianiste Alfred Cortot, travaillé par des ambitions politiques effarantes, l'historien de la musique Gustave Samazeuilh, suspecté - à juste titre - d'intelligence avec l'occupant à partir de 1940 et le chef d'orchestre Hans Knappertsbusch, l'un des loups hurlant contre Thomas Mann au début de 1933. L'auteur de La Montagne magique s'était alors inquiété de la collusion à venir entre l'œuvre de Wagner et le nazisme. Le même Knappertsbusch sera au nombre des bêtes noires de Wieland Wagner, se heurtant régulièrement à lui quand ils seront contraints de collaborer au Festival de Bayreuth. Quant à sa mère Winifred, elle fait état de l'aversion de l'aîné de ses fils à l'égard du célèbre Kapellmeister dans sa lettre du 10 septembre 1963 à Paul Boulet. On imagine l'ancienne amie intime d'Hitler, une cigarette aux lèvres, dactylographier elle-même cette correspondance destinée à un Français maîtrisant manifestement la langue allemande et nostalgique comme elle - du statu quo ante. L'attitude de Boulet à l'égard de Winifred n'est plus, alors, teintée de distance respectueuse. Il s'implique dans les querelles familiales se déroulant à Wahnfried 9 . Lui rendant visite en août 1965, le Français lui fait remettre - au préalable - un bouquet de fleurs et un carré de soie Hermès. Paul Boulet compare désormais Wieland à Fafner et Wolfgang à Fasolt ${ }^{10}$. Il le fait avec l'approbation de leur mère, celleci soulignant - le 18 août 1964 - que seul Wolfgang est capable de bien mettre en scène la Tétralogie. Depuis, l'Histoire aura opposé un cinglant démenti aux affirmations de Winifred!

Les énervements de l'été 1963, suscités, on l'a vu, par la nouvelle mise en scène de Wieland pour Les Maîtres chanteurs de Nuremberg, seront l'occasion pour Boulet de prédire la fin de Bayreuth. Sous ce rapport, sa lettre du 7 septembre de la même année à Winifred prouve que, vus à l'aune du monde actuel, ces propos n'ont rien de nouveau. Ils montrent toujours la distinction existant entre 
la façon dont des amateurs passionnés d'art lyrique imaginent la représentation concrète des ouvrages de leurs compositeurs-fétiches et le travail des metteurs en scène. Pourtant, un art qui ne se renouvelle pas est condamné à péricliter. Paul Boulet voulait-il garder les trésors wagnériens pour lui ? La question reste ouverte. Quoi qu'il en soit, il n'était en tout cas ni le premier ni le dernier à camper sur de telles positions...

Le texte de Wieland, dont nous reproduisons ici le tapuscrit, était accompagné d'un mot signé par J. Taut, probablement sa secrétaire, qui signifiait à Boulet son envoi joint à la lettre citée plus haut. Le traducteur de cette version française n'y est par contre pas mentionné, et est resté inconnu.

\section{Philippe Olivier}

\section{Notes}

1 - Richard Wagner est né à Leipzig le 22 mai 1813.

2 - Lettre d'Henri Sauguet à Paul Boulet du 17 janvier 1959 (fonds Boulet-Devraigne)

3 - Lettre de Paul Boulet à Wieland Wagner du 26 août 1951 (fonds Boulet-Devraigne)

4 - Lettre de Paul Boulet à Winifred Wagner du 19 octobre 1966 (fonds Boulet-Devraigne)

5 - Lettre de Paul Boulet à Winifred Wagner du 16 septembre 1963 (fonds Boulet-Devraigne)

6 - Richard Wagner und das neue Bayreuth / herausgegeben von Wieland Wagner. - München : List, 1962

7 - Il s'agit de La mise en scène du drame wagnérien.

8 - Le même reproche avait été formulé, dès 1876, à Richard Wagner lui-même!

9 - Lettre de Paul Boulet à Winifred Wagner du 8 janvier 1965 (fonds Boulet-Devraigne)

10 - Ces personnages de L'Or du Rhin sont mus par une rivalité sans limites pour s'emparer du pouvoir des dieux sur l'humanité. Au cours du même ouvrage, Fafner assassine son frère Fasolt. Une pareille référence est utilisée par Paul Boulet dans sa lettre à Winifred du 12 avril 1964. Ce courrier est également conservé à la Bibliothèque nationale et universitaire de Strasbourg. 
Logique, prêchée ex-cethedra, de son dogze de I'authent1que rlál1té a I'oeuvre; ces théories néfastes pour 1 'exclstence nême đu théatre n'étant A vral dire réalisables qu'appuýós per des mesures de police. Wéanmolns elles servent aujourd hul d'argument de base pour les partisans de le fldélité a l'oeuvre.

Les annotations scénlques qu1, apparemuent, posent de s1 lourds problenes, ne sont en falt rien d'autre que des ina1cations annexes, A I'intention de lecteurs ne connalssant pas Ia pertition - car, si l'on a lo courage d'examiner les part1tions selon les paroles mêties de Wegner, "avent d'attelndre leur but, qui est de servlr de nodele poir flxer techniquement une représentation parfaite, elles demeurent une éniere pour In critique esthetique, "ies "hiéroglyphes de la mimique" et des "objete de le fantalsie oréatrice" cowie dit Paul Bekcker. Selon xocondax Wagner les notes et les veleurs n'ont qu'tune slgniflcation indirecte en tant que forces au service de la mimique. Les 1ndiestions sciniques ne seralent done que des points de repèro pour les lecteurs du texte, des accessolres "đonnés par-cessus le narchi" par le compositeur, pour ouvrir d'autres horizons a la fantnisie par I'Intermédalre d'une fonction l1ttéraire.

Les 1dées de Wegner para1saont alanétralenent opnosées à celles de Pittzner.

Du vivant de Wagner ses visions théatrales fusent primitiverient wontíos en s'ineplrant des tableaux de genre du style uederteler, pu1s dans le golt romantique attardé des paysages "naturalistes" et de la peinture ilatorlque. Ses Incications de rise en scane furent rósiliśles solt A la cadence des riouvements stéréotypás de L'opéra 1telien, solt avec le milique pathét1que des leintnger. Les tsnolenages de x'́poque qui nous

rapportent ee cue Je conpositeux pensalt du style et do la 
technique de ses contemporains sont fort variés : 11s passent de 1'enthousiasme â le réserve discrate ou a la rósignation impussante: . Iaponstatation que ses mises en scène éta1ent "gênées per le style de son 6́poque " se maint1ent - avec certaines rúserves - et ne signtele sucu amoindrissement de s8 vision thestrale. I $n^{\prime} y$ s pour Ie théatre qui v1t d'autre style que Ie "style de l'époque" - quitte a ce que les générations futures traucuncx le trouvent plein C'1nsupportables clichés ou qu'ellea ne le tiennent pour grandiose et g'en Insplrent .

Hais c'est une insulte Wagner en tant que compositeur d'1dentifier son oeuvre mythique ovec la notolre nédiocrité et le psendo-naturalisme fipulssent de la peinture alletiande de . son époque quí, sans 1dée personnelle, tente de vulgariser les scquisitions du classicisme et du romantisme selon le gout d'une bourgeo1s1e enrichie.

L'autorlté sans pareilule de Rlcherd Wegner at, d'autre part, I'. spoct nógatif de tout I'art théatral on, pinz próclsément 1e ranque de temps, d'argent, Ie routine et I'1 gnorance, les vnriations de "modßles" surchargés de déta1ls de catte époque sens 1mportance art1stique, se trouventy de nos fours 4 Être encore tout naturellement acceptós dans 10 monde ent1er. Cos modales collent corrs du sirop a l'oeuvre wagnórienne, troc 1 u1 conférent un caractère "poussiéreux" mêne dens des représentations nontbes par đes mótropoles dítes d'avant-garde.

D'audecienx essa1s pour s'affranch1r de l'obsissance aveugle envers la trsdition forite - et mettre los 6í́nents formels du

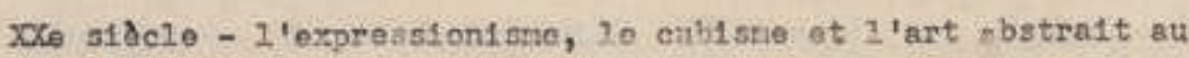
servico de 1 'oeuvre de Wagner - n'ont 6t6 tentés qu'en fletuagne et en Sulsse, f́tent d'allieurs fort ortiqunés ot soupgonnés de tendences politiques. Le ceractore trefique de cette oenvre no convient pas au style góntal A'Asplas ot los courereux essals 
de Klenperer et de Fehling sont demeurés sens sulte en raison de In tournure prise par la politique vers 1930, ce quil les empêche de servir de modale en dehors de Berlin. Bt Beyreuth, de son cóté ne commenģs qu'en 1927 , non sans hés1tations, A chercher un moyen terme qui concllie la conception d'Applas et celle des part1sans de la fidél1té absolue . Cosime Wegner,en bannissant I'ouvrage d'Applas "la Musleue et la Wise en Scene", maintint a Bryreuth pendant des diza1nes d'années f une tendance artist1que depuls longterps dépassée dont I'erfet éta1t diamétralement opposé ses propres intentions révolutionna1res. In vole ou s'engkgea Holnz Tletfen de 1933 \& 1945 pour ses mises en scòne đe Bayreuth sous I'ère du "natural1sme de I'état", avoc I'opposition 1rritée des vieux wagnériens, ces gardiens du Groal elors encore influents, mérite non seulement qu'on lu1 rende Justice sur ce point historlque ma1s qu'on is mentionne tout particulidrement.

I* question théorique si volontlers posée, de savo1r quelle aura1t été la róretion de Wagner "s'11 viva1t de nos joura" devant une mise en scene solt disant moderne, est aussi superflue que de savolr ce que Beethoven diralt de 1'interpabtation de sa neuvieme symphonle par Toscantn1, L'avis de Vozart au sujet du style de ses oeuvres telles qu'on les donne a Salzbourg, ou 1 'opinion de Goethe A propos de son "Faust" mis en scene par Gustav Grunfens a Hamboure - Bn matidare d'art seul cospte le temps présent ot le Ileu actuel d 'aujourd hu1. Impossible d'accepter pour I'oeuvre wagnórienne une lo1 d'exception qu1 la tienne en dehors de I'évolution inóvitable de I'h1stoire de l'art et de la pensée.

"Que nous al gniflent les 1déos d'arrogants cécoratenrs ou de metteurs en soène en quête de publicité - on ne retouche pas un I1tien ou un Direr sans compettre un sacrildge" ( et quel 


\section{$-5-$}

we gnérien pleceralt I'oeuvre de son "maftre" A un rang inférleur a celu1 des neftres de 28 peinture clessique ?). Yo1c1 ce qui, depuis.1951, est consterment d1scutế, sous de dirférentes fortes, s'eppryant sur les stupldes arguments de l'orthodoxle wagnérienne contemporaine qui ne deviennent pes plus persuasifs du falt qu'ils sont opinffitrement répétés : le drane et le tebleau sont des réslités bien différentes et fort peu cołparables ...

Vals I'ensemble de l'oeuvre "romentique" do Wegner ne réclame-t-elle pas - du moins le prétend-on - un décor fiadla A Le ról1té, 1'12zusion et la fidólité historique ? Me detande-toelle pas au moins la coordination du peysege a l'ansemble dest arts ?

L'utople wagnór1onne, de I/oeuvse d'art de I'evonich, a toujours été une nbomination du point de vue 11ttórelre et artisticue .

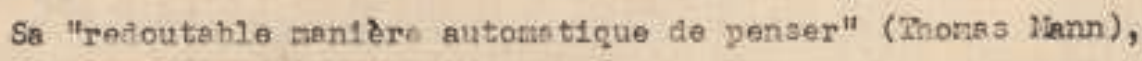
conault a1sóment, depuls Metzsohe, tont Wegrer a $L^{\prime}$ ebsurde. Cons1dórá histor1quement le "Gesantkunstwer)c" , cette synthase des arts, est la formilo Individuelie trouvío par Wagner pour

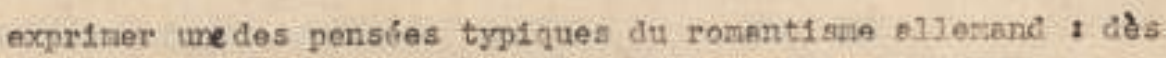
1800 , Hercer le prét1salt nythiquesent et Novel1s, Schelling,

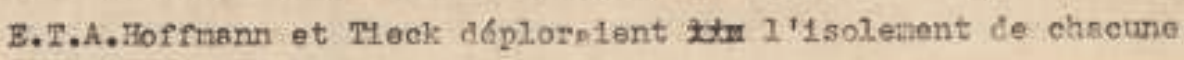
des brancies de l'art. Tous pecherchent un "posnt comaun, come I'bteit Is rythologie de I'intinulteil et désirent nrderaent

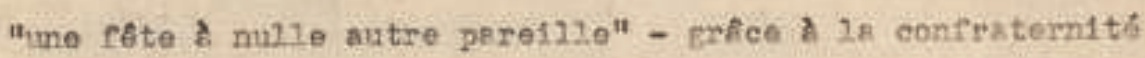

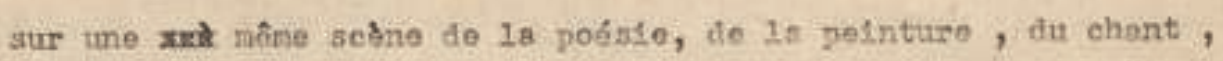

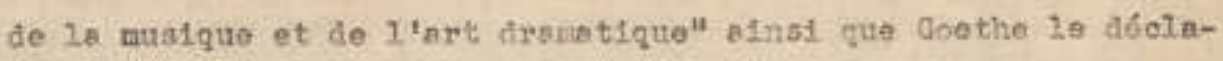
rait \& Hoikermann on taers 1225 .

Is reit, de la part de Wagner, de créer uno oeuvre sul donne

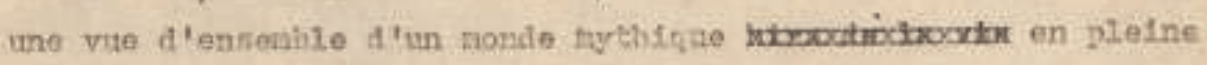
ofvilsstion technique ot rntioneliste roghtarnte the force 
créstrice Incom ensurable.

les réellsations pratioues

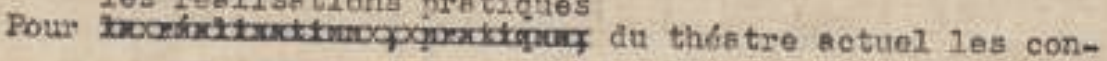
ceptions fort délavies de Wegner ne si gnifient guere davantage que sea rérlexions sur lo vógáterismo on ropht et th Climat" . Quel deplorable nalontendu de falre dícouler de la théorle du "Gesanticunstweri" un cogme sty1.stique, uno 201 et uno recette poir Ie theatre et, avec des prótentions $\lambda$ 1'1nfa1111billté, de s'blever, au nom de le sauvegarde des prinolpes, contre toute plse on scine qui n'est polnt disposíe a confondre lo notion essentielle du romentisne avec celle du naturaliane đu sidcle dernier.

Wagner, Ini-Eêne, perlera plus tard du "malneureux" "Gesamtkunstwerk" . Ce n'est done pas un ssor118ge 31 , de nos fours, $I^{\prime}$ on ne prolonge pas cette erreur, vra1sembleblement nécessaire f au temps ol Wagner la penselt .

Une ulse on scone de 1 'oeuvre wagnórienne ut111 sant des procédís chorberaphiques at des principes qu1 tontent d'assuJottir Les art1stes Iyrigues aux ragles dranat1ques, se servant de la lunilare conve moyen đramatique ot proment los bliments đócoratifs de I'art contorgora1n - "alenou" v1s1onia1res, w00x 7xx abstraction géomótrique, couleurs et forres syriboliquez pour créer l'espace, n'est par forcérent un beliet", un "musicpl", un "oratorio", me "revue", un "concert wagnorien ", "Qrérícan1sée", "prolátar1sée", "bolchev1sée" ou nôno - horr1blenent d1t - un "strawinsk". Des mote d'esprit ansol faclles qui, pour caractóriser des mises en scène nocernes pour les détru1re, associont Ie notion "ent1rosiantigue" et " nti-nI:emande" a Ia vie politique et Intellectuelle de nos fours afin d'attelnare plus aisément les nréjugés de l'anciteur hulvino 
1'smuzent Incontestahloment . Nale des bons nots aussil sinnlistes ne peluvent gu'efeleurer lo problome de l'sdeptetion de 1'oeuvse car $11 \mathrm{~s}$ prótendent, A la légère, rendre absolue une forme de mise en scène dónodß́e. Wottre en scène n'ast pas uniquezent I'Brt de reprodulire $t$ cela ne sert la erétion dronatique qu'en se rejeunissant constamient .

Les probldmes des oouvros wagnoriennes demeurent de tout temps valables ear 11 s traitent A'éternels probldres humeins. Cependant los Indications do Wagnor relatives nux décors ot क la nise en scene ne correspondent qu'au théatre du XDVe s1əcle. Leur rélis tion fiddle, si nêne elle nut fanals être réalisable, n'est plus le eritérlut d'une $m 1$ so on scene sctuelle. Lu 11eu d'un dfeor atérile a la nodo du s18ole cernier et d'ancionnes Indleations de motivenent, 2 'on tenters de $\rightarrow$ lallsor sur sedno I'oouvre arch1 typlque de Wagner selon 3.0 espactore de notre 6́porue ce qui ne peut rbussir gue per the fecrintion apirituelled osant "renonter zux sources nônie! de 1'oenvre". De IA devra-telle etre refalte, toufours : norveru - en dícisecrent des hili-

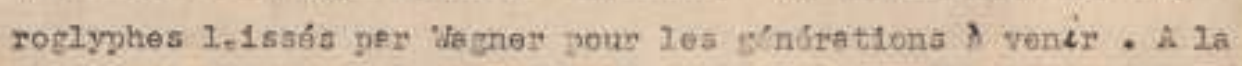
In de In prenilare représentetion les Whaftres Chertsurat A

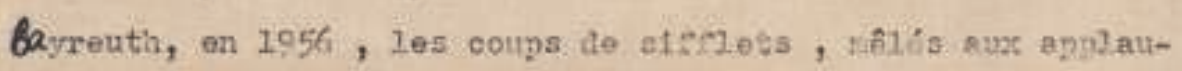

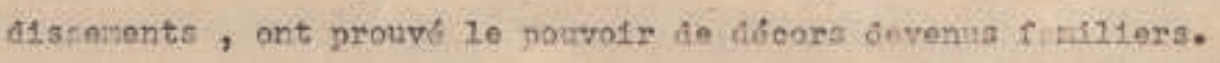
Ces coups de s1rc2nts ne eicentrient psis I'oeuvre in Wsener,

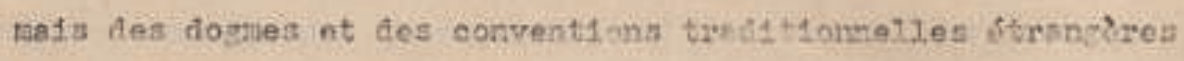
A It vio thistrale actue?le. 